\title{
Similarity Solutions for Laminar Boundary Layer Flow of Darcian Fluid over Horizontal Plate with a Convective Boundary Condition
}

\author{
Rama Charan Chaudhary ${ }^{1^{*}}$, Gopal Naraian Purohit ${ }^{2}$, Preeti Garg ${ }^{3 *}$ \\ ${ }^{1}$ Department of Mathematics, University of Rajasthan, Jaipur, India \\ ${ }^{2}$ Department of Mathematics, Banasthali Vidyapith, Banasthali, India \\ ${ }^{3}$ Department of Mathematics, Kirori Mal College, University of Delhi, New Delhi, India \\ Email: *rcchaudhary@rediffmail.com,gn_purohitjaipur@yahoo.co.in, "preetigarg152@gmail.com
}

Received 20 January 2016; accepted 26 February 2016; published 29 February 2016

Copyright (C) 2016 by authors and Scientific Research Publishing Inc.

This work is licensed under the Creative Commons Attribution International License (CC BY).

http://creativecommons.org/licenses/by/4.0/

(c) (i) Open Access

\section{Abstract}

This paper considers the problem of hydrodynamics and thermal boundary layers of Darcy flow over horizontal surface embedded in a porous medium. The solutions of such problems for the cases of uniform surface temperature and variable surface temperature have been studied and analysed in many papers. This paper, however, attempts to find similarity solutions for the Darcy flow problem with a convective boundary condition at the plate surface. It is found that the solution is possible when the heat transfer coefficient is proportional to $x^{-2 / 3}$. The numerical solutions thus obtained are analyzed for a range of values of the parameter characterizing the hot fluid convection process. Analytical expressions are provided for local surface heat flux and total surface heat transfer rate while the flow variables are discussed graphically.

\section{Keywords}

Similarity Solution, Horizontal Plate, Heat Transfer Rate, Darcy Flow, Convective Boundary Condition

\section{Introduction}

The study of natural convection flow and heat transfer in saturated porous medium from surfaces which are at a temperature different from the surrounding medium has always been a topic of great interest due to several engineering and geophysical applications. An extensive study of convection flow in porous media is provided by

${ }^{*}$ Corresponding authors.

How to cite this paper: Chaudhary, R.C., Purohit, G.N. and Garg, P. (2016) Similarity Solutions for Laminar Boundary Layer Flow of Darcian Fluid over Horizontal Plate with a Convective Boundary Condition. Applied Mathematics, 7, 313-319. 
Nield and Bejan [1], Ingham and Pop [2] and Vafai [3].

The similarity solutions for many different problems of free convection fluid flow in saturated porous medium have been obtained and analyzed by various authors. Cheng and Chang [4] studied free convection in a saturated porous medium over a horizontal impermeable surface where wall temperature is a power function of distance from the origin. Cheng and Minkowycz [5] studied the same problem for the flow over a vertical impermeable plate. Mealey and Merkin [6] and Postelnicu and Pop [7] extended the problem and analysed the flow in the presence of internal heat generation. Heat transfer analysis for free convection along horizontal flat plate with a variable wall temperature in a porous medium saturated with non-Newtonian fluid was studied by Gorla and Kumari [8]. Solutions and results for flow across heated curved surfaces and arbitrary shaped bodies are also available in many papers-Grosan et al. [9], Nakayama and Koyama [10], Bagai and Nishad [11] to mention a few.

In all the papers cited above, the problems are solved when the flow is driven either by a prescribed surface temperature or by a prescribed heat flux. Here, in this paper, a somewhat different driving mechanism for a free convection along the surface is considered, where it is assumed that the flow is also set up by the convective heating of the surface. Aziz [12] used this type of convective boundary condition to study the classical boundary layer flow over a flat plate. It has since been used by many other authors, for example Makinde [13] and Olanrewaju et al. [14], for studying several natural convection problems with various physical aspects.

However these convection problems constitute a relatively new and active area in the field of convection in porous media. To the best of author's knowledge, no such solution has been attempted for the Darcy flow in porous medium with a convective surface boundary condition. Hence, in the present study the problem of laminar boundary layers over flat plate in Darcy regime with a convective boundary condition has been considered. The paper demonstrates that a similarity solution is possible if the convective heat transfer of the fluid heating the plate on its lower surface is proportional to $x^{-2 / 3}$. Numerical values of the resulting similarity solutions are provided for a range of values of the parameter $a$, which characterizes the hot fluid convection process.

\section{Mathematical Formulation of the Problem}

We consider the problem of hydrodynamics and thermal boundary layer flow over a flat plate embedded in a porous medium saturated with Newtonian fluid. The momentum and thermal boundary layers exist along horizontal surface whenever wall temperature differs from that of the surrounding fluid. We take $x$-axis along the plate and $y$-axis along the normal to the plate. The flow is based on the following assumptions:

1) two-dimensional flow,

2) the flow is assumed to be slow enough to conform to the Darcy regime i.e. Darcy flow model,

3) total thermodynamic equilibrium between the surfaces,

4) negligible viscous dissipation,

5) isotropic porous medium and

6) constant thermo-physical properties, with the exception of the assumed linear relation between density and temperature in the buoyancy term (the Oberbeck-Boussinesq approximation).

Then, under boundary layer approximations, the equations governing the flow as given by Cheng and Chang [4] are

$$
\begin{aligned}
& \frac{\partial u}{\partial x}+\frac{\partial v}{\partial y}=0 \\
& \frac{\partial u}{\partial y}=-\frac{K \rho_{\infty} g \beta}{\mu} \frac{\partial T}{\partial x} \\
& u \frac{\partial T}{\partial x}+v \frac{\partial T}{\partial y}=\alpha \frac{\partial^{2} T}{\partial y^{2}}
\end{aligned}
$$

where $u$ and $v$ are the velocity components in the horizontal and vertical directions respectively. $\rho, \mu, \beta$ are the density, viscosity and the thermal expansion coefficient of the convective fluid. $K$ is the permeability of the porous medium, $\alpha=k / \rho C_{p}$ is the equivalent thermal diffusibility with $k$ denoting the thermal conductivity of the saturated porous medium and $C_{p}$ is the specific heat at constant pressure of the convective fluid. $T, p$ and $g$ are the temperature, pressure and gravitational acceleration respectively. The subscript $\infty$ refers to the condi- 
tion at infinity.

In this paper we assume that the bottom surface of the plate is heated by convection from a hot fluid at constant temperature $T_{f}$ which provides the heat transfer coefficient $h_{f}$. Boundary conditions at the plate surface and far into the colder fluid are given by Aziz [12] and may be written as

$$
\begin{aligned}
& v=0,-k \frac{\partial T}{\partial y}=h_{f}\left(T_{f}-T(x, 0)\right) \text { at } y=0 \\
& u \rightarrow 0, T \rightarrow T_{\infty} \text { as } y \rightarrow \infty
\end{aligned}
$$

To determine similarity solutions to momentum and energy equations coupled with boundary conditions (4) and (5), we introduce the following dimensionless variables

$$
\eta=\frac{y}{x}\left(R a_{x}\right)^{1 / 3}, \psi=\alpha\left(R a_{x}\right)^{1 / 3} f(\eta), \theta=\frac{T-T_{\infty}}{T_{f}-T_{\infty}}
$$

where $\psi$ is the stream function such that $u=\frac{\partial \psi}{\partial y}$ and $v=-\frac{\partial \psi}{\partial x}$ and $R a_{x}=\frac{K \rho_{\infty} g \beta}{\alpha \mu}\left(T_{f}-T_{\infty}\right) x$ is the modified local Rayleigh number.

In terms of the new variables, it can be shown that the velocity components are given by

$$
\begin{aligned}
& u=\frac{\alpha}{x}\left(R a_{x}\right)^{2 / 3} f^{\prime} \\
& v=\frac{2 \alpha y}{3 x^{2}}\left(R a_{x}\right)^{2 / 3} f^{\prime}-\frac{\alpha}{3 x}\left(R a_{x}\right)^{1 / 3} f
\end{aligned}
$$

and the governing equations become

$$
\begin{aligned}
& f^{\prime \prime}-\frac{2}{3} \eta \theta^{\prime}=0 \\
& \theta^{\prime \prime}+\frac{1}{3} f \theta^{\prime}=0
\end{aligned}
$$

coupled with boundary conditions

$$
\begin{aligned}
& f(0)=0, \quad \theta^{\prime}(0)=-a[1-\theta(0)] \\
& f^{\prime}(\infty)=0, \theta(\infty)=0
\end{aligned}
$$

where $a=\frac{h_{f}\left(R a_{x}\right)^{-1 / 3}}{k} x$

The primes in Equations (8)-(11) denote differentiation with respect to $\eta$.

For momentum and energy equations to have a similarity solution, the parameter $a$ must be constant and not a function of $x$. This condition is met if the heat transfer coefficient $h_{f}$ is proportional to $x^{-2 / 3}$.

Therefore, we assume that

$$
h_{f}=c x^{-2 / 3} \text {, }
$$

where $c$ is a constant.

With these assumptions we have

$$
a=\frac{c}{k}\left(\frac{K \rho_{\infty} g \beta}{\alpha \mu}\left(T_{f}-T_{\infty}\right)\right)^{-1 / 3}
$$

With $a$ as defined by Equation (12), the solutions generated are the local similarity solutions.

\section{Results and Discussions}

Equations (8) and (9) are coupled non-linear differential equations for $f$ and $\theta$ with boundary conditions given by 
(10) and (11) where $a$ is defined by (12). Numerical solutions to these coupled non-linear boundary value problem can be obtained by using shooting method technique. We first convert the boundary value problem to an initial value problem and make a systematic guessing of slopes at $\eta=0$. The asymptotic boundary condition at $\eta=\infty$ is replaced by $\eta=10$ as is the standard practice in the boundary layer analysis.

The graphs for $f, f^{\prime}, \theta, \theta^{\prime}$ vs $\eta$ are shown in Figures 1-4 respectively for certain values of the parameter $a$. The numerical computations of the plate surface temperature $\theta(0)$ and the local Nusselt number which is proportional to $\left[\theta^{\prime}(0)\right]$ are worked out and their numerical values are presented in Table 1 . It should be noted that the boundary layer approximations used in the analysis are valid if convection takes place in a thin layer around the heating surface.

From Equation (6), it can be seen that $u \rightarrow 0$ outside the momentum boundary layer. We notice from Figure 1 that the value of the dimensionless stream function $f$, as $\eta \rightarrow \infty$, is positive and finite and thus, the value of the vertical velocity $v$ outside boundary layer is, in general, not zero. This can also be seen from the equation $v_{\infty}=-\frac{\alpha}{3 x}\left(R a_{x}\right)^{\frac{1}{3}} f(\infty)$.

The values of the dimensionless velocity $f^{\prime}=\frac{u}{u_{r}}$, where $u_{r}=\frac{\alpha}{x}\left(R a_{x}\right)^{2 / 3}$, plotted against $\eta$ in Figure 2 reveal that for each value of $a$ the value of $f^{\prime}$ decreases as $\eta$ increases.

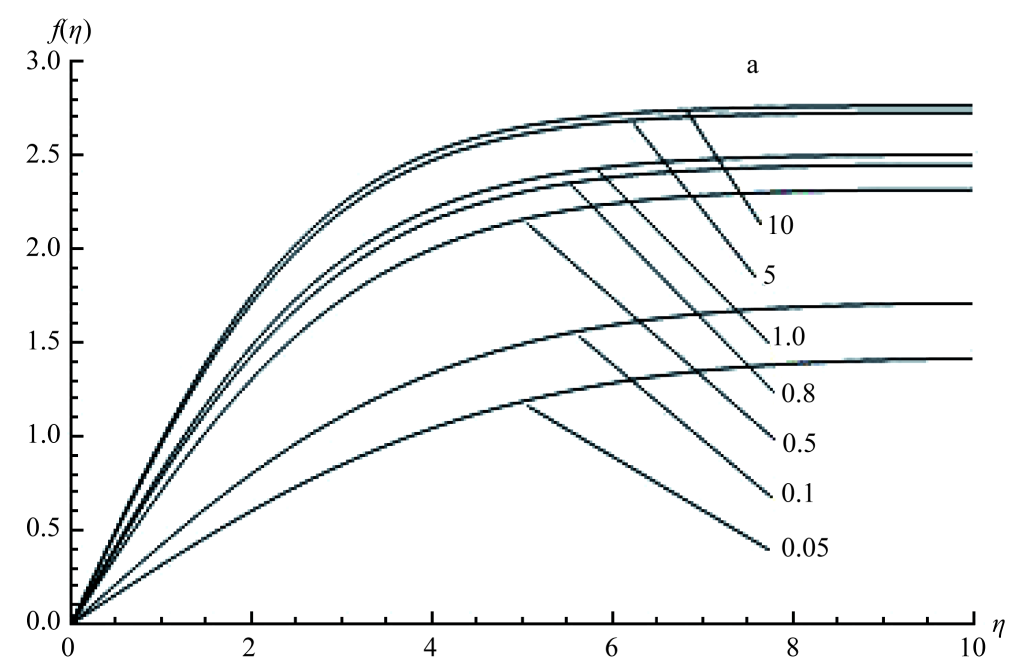

Figure 1. Graph of $f$ vs $\eta$ for various values of the parameter $a$.

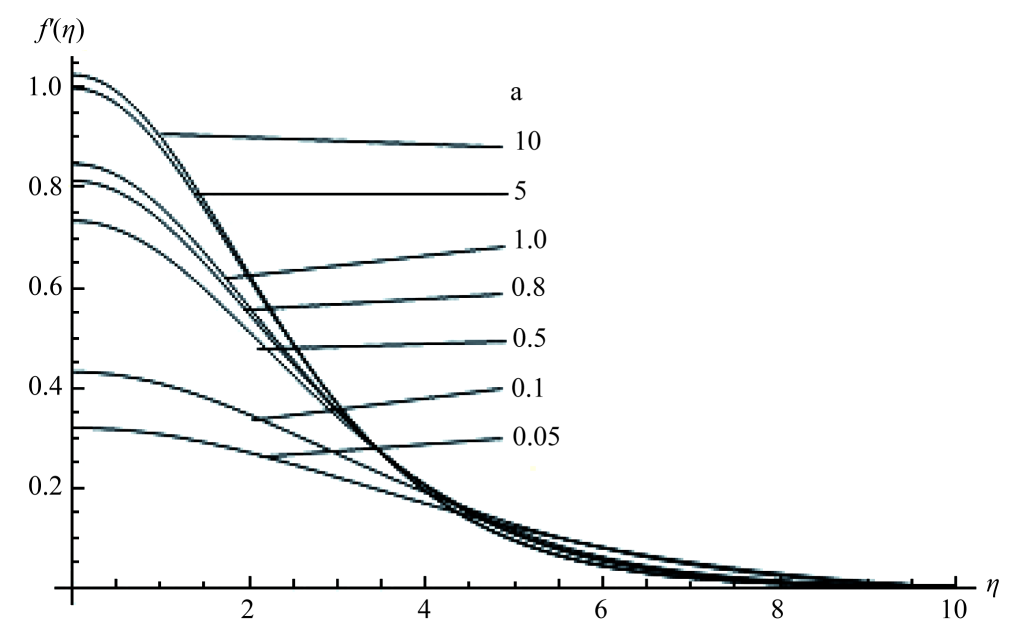

Figure 2. Velocity distribution for various values of the parameter $a$. 


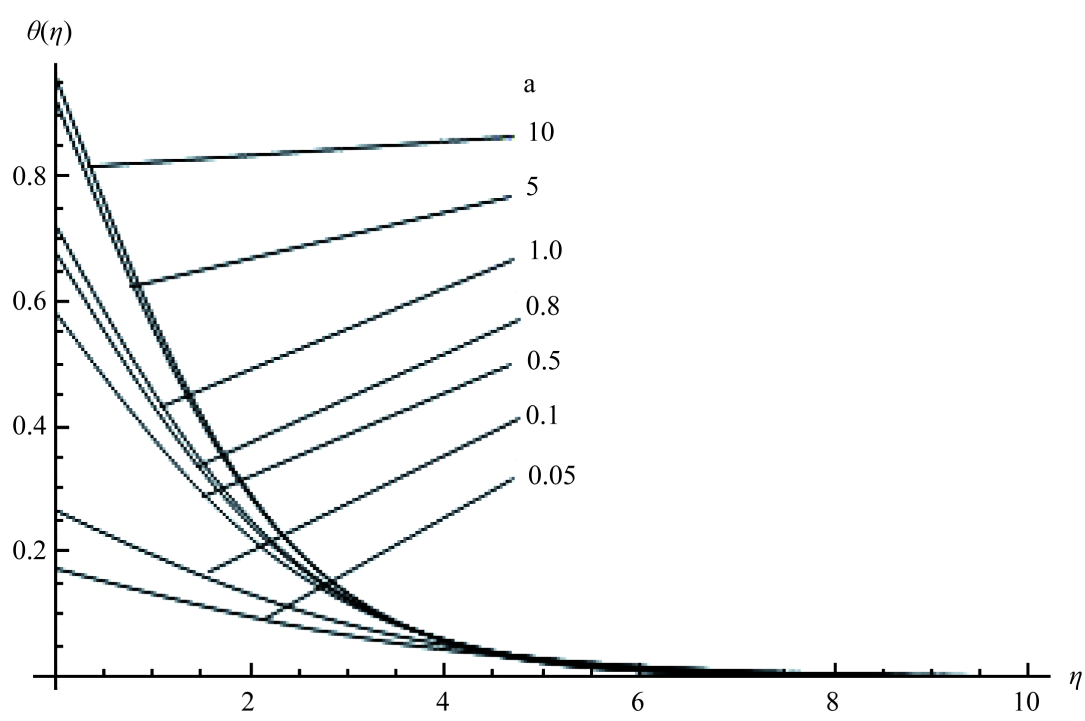

Figure 3. Temperature distribution for various values of the parameter $a$.

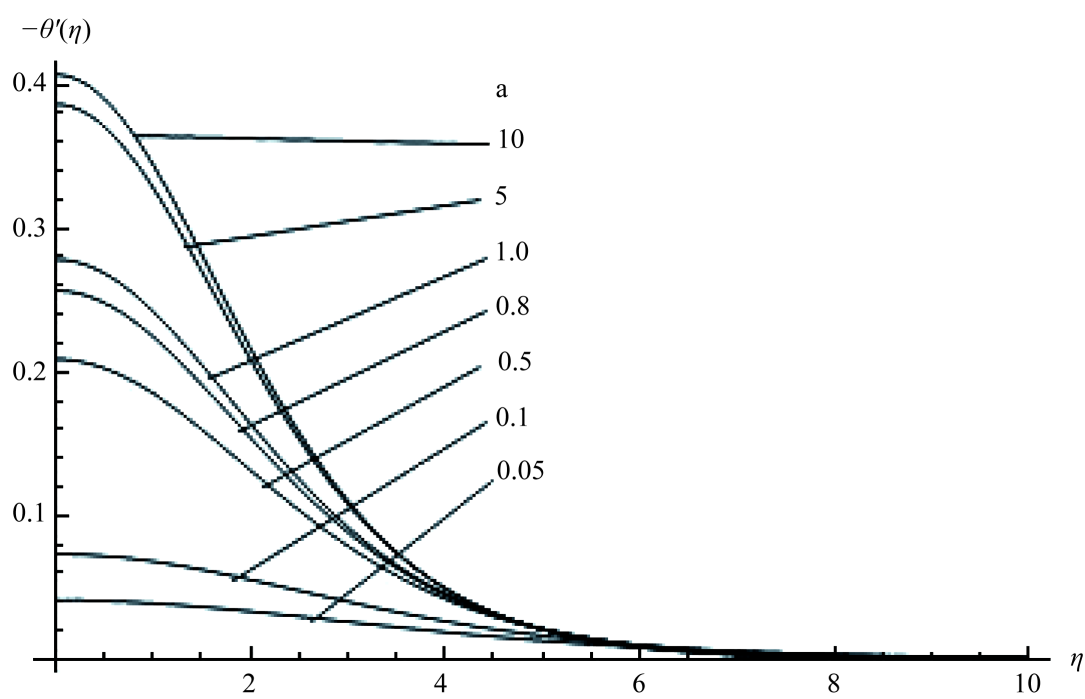

Figure 4. Graph of $\left(-\theta^{\prime}\right)$ vs. $\eta$ for various values of the parameter $a$.

Table 1. Values of $\theta(0)$ and $\left[-\theta^{\prime}(0)\right]$ for different values of the parameter $a$.

\begin{tabular}{ccc}
\hline$a$ & $\theta(0)$ & $-\theta^{\prime}(0)$ \\
\hline 0.05 & 0.173110 & 0.041344 \\
0.1 & 0.266035 & 0.073396 \\
0.5 & 0.582140 & 0.208930 \\
0.8 & 0.679166 & 0.256667 \\
1.0 & 0.721672 & 0.278328 \\
5.0 & 0.922731 & 0.386346 \\
10.0 & 0.959309 & 0.406912 \\
\hline
\end{tabular}


Table 1 provides numerical values of $\theta(0)$ and $\left[-\theta^{\prime}(0)\right]$ from which it is clear that both surface wall temperature and the heat transfer rate along the surface of the plate increases as $a$ increases. It is to be noted that for fixed cold fluid properties $a$ is directly proportional to the heat transfer coefficient $h_{f}$ at any location $x$ and the thermal resistance on the hot fluid side is inversely proportional to $h_{f}$. Therefore when $a$ increases the convection resistance decreases on the hot fluid side and as a result the surface temperature increases as exhibited in Figure 3 and in Table 1. It is also shown in Figure 3 that maximum value of dimensionless temperature at $\eta=0$ is 1 and its value decreases as $\eta$ increases.

The local surface heat flux can be computed from $q=-k\left(\frac{\partial T}{\partial y}\right)_{y=0}$

In terms of dimensionless temperature $\theta$, this can be rewritten as

$$
q(x)=k\left(\frac{K \rho_{\infty} g \beta}{\alpha \mu}\right)^{1 / 3}\left(T_{f}-T_{\infty}\right)^{4 / 3} x^{-2 / 3}\left[-\theta^{\prime}(0)\right]
$$

The total surface heat-transfer rate for a surface with a length $l$ and a width $w$ can be computed from

$$
Q=w \int_{0}^{l} q(x) \mathrm{d} x
$$

which gives

$$
Q=-3 k w\left(\frac{K \rho_{\infty} g \beta l}{\alpha \mu}\right)^{1 / 3}\left(T_{f}-T_{\infty}\right)^{4 / 3} \theta^{\prime}(0),
$$

when $a$ is constant and

$$
Q=-k w\left(\frac{K \rho_{\infty} g \beta}{\alpha \mu}\right)^{1 / 3}\left(T_{f}-T_{\infty}\right)^{4 / 3} \int_{0}^{l} x^{-2 / 3} \theta^{\prime}(0) \mathrm{d} x
$$

when $a$ is a function of $x$.

This is because when $a$ is constant the surface temperature $\theta^{\prime}(0)$ is independent of $x$.

\section{Conclusion}

It is found that under boundary layer approximations, a similarity solution for the Darcy flow over a flat plate embedded in a porous medium with a convective boundary condition at the plate surface is possible if the convective heat transfer of the fluid heating the plate on its lower surface is proportional to $x^{-2 / 3}$. The numerical solutions of the resulting thermal similarity equations provided in the table can be used in the expressions for total surface heat flux to get an approximate estimate of energy transfer rate between the horizontal surface to the surrounding saturated porous medium.

\section{References}

[1] Nield, D.A. and Bejan, A. (2013) Convection in Porous Media. 4th Edition, Springer, New York. http://dx.doi.org/10.1007/978-1-4614-5541-7

[2] Ingham, D.B. and Pop, I., Eds. (2005) Transport Phenomena in Porous Media III. Elsevier, Oxford.

[3] Vafai, K., Ed. (2015) Handbook of Porous Media. 3rd Edition, CRC Press, Taylor and Francis Group, Boca Raton, Florida.

[4] Cheng, P. and Chang, I. (1976) Buoyancy Induced Flows in a Saturated Porous Medium Adjacent to Impermeable Horizontal Surfaces. International Journal of Heat and Mass Transfer, 19, 1267-1272. http://dx.doi.org/10.1016/0017-9310(76)90078-8

[5] Cheng, P. and Minkowycz, W.J. (1977) Free Convection about a Vertical Flat Plate Embedded in a Porous Medium with Application to Heat Transfer from a Dike. Journal of Geophysical Research, 82, 2040-2044. http://dx.doi.org/10.1029/JB082i014p02040

[6] Mealey, I. and Merkin, J.H. (2008) Free Convection Boundary Layers on a Vertical Surface in a Heat Generating Porous Medium. IMA Journal of Applied Mathematics, 73, 231-253. http://dx.doi.org/10.1093/imamat/hxm048 
[7] Postelnicu, A. and Pop, I. (1999) Similarity Solution of Free Convection Boundary Layers over Vertical and Horizontal Surfaces in Porous Media with Internal Heat Generation. International Communications in Heat and Mass Transfer, 26, 1183-1191. http://dx.doi.org/10.1016/S0735-1933(99)00108-6

[8] Gorla, R.S.R and Kumari, M. (2003) Free Convection in Non-Newtonian Fluid along a Horizontal Plate in a Porous Medium. Heat and Mass Transfer, 39, 101-106.

[9] Grosan, T., Postelnicu, A. and Pop, I. (2004) Free Convection Boundary Layer over a Vertical Cone in a Non-Newtonian Fluid-Saturated Porous Medium with Internal Heat Generation. Technische Mechanik, 24, 91-104.

[10] Nakayama, A. and Koyama, H. (1987) Free Convective Heat Transfer over a Non-Isothermal Body of Arbitrary Shape Embedded in a Fluid-Saturated Porous Medium. Journal of Heat Transfer, 109, 125-130. http://dx.doi.org/10.1115/1.3248032

[11] Bagai, S. and Nishad, C. (2012) Effect of Variable Viscosity on Free Convection Heat Transfer over a Non-Isothermal Body of Arbitrary Shape in a Non-Newtonian Fluid Saturated Porous Medium with Internal Heat Generation. Transport in Porous Media, 94, 277-288. http://dx.doi.org/10.1007/s11242-012-0004-2

[12] Aziz, A. (2009) A Similarity Solution for Laminar Thermal Boundary Layer over a Flat Plate with a Convective Surface Boundary Condition. Communications in Nonlinear Science and Numerical Simulation, 14, 1064-1068. http://dx.doi.org/10.1016/j.cnsns.2008.05.003

[13] Makinde, O.D. (2012) Effect of Variable Viscosity on Thermal Boundary Layer over a Permeable Flat Plate with Radiation and a Convective Surface Boundary Condition. Journal of Mechanical Science and Technology, 26, 1615-1622. http://dx.doi.org/10.1007/s12206-012-0302-1

[14] Olanrewaju, P., Anake, T., Arulogun, O.T. and Ajadi, D. (2012) Further Results on the Effects of Variable Viscosity and Magnetic Field on Flow and Heat Transfer to a Continuous Flat Plate in the Presence of Heat Generation and Radiation with a Convective Boundary Condition. American Journal of Computational and Applied Mathematics, 2, 42-48. http://dx.doi.org/10.5923/j.ajcam.20120202.08 